\title{
Purpuric drug eruption without leukocytoclastic vasculitis associated with vancomycin
}

\author{
Tamaki Nakamura, ${ }^{1}$ Hiroyuki Wakiguchi, ${ }^{1}$ Fumiko Okazaki, ${ }^{1}$ Nobuyuki Asano, ${ }^{2}$ Yoshinobu Hoshii, ${ }^{3}$ Shunji Hasegawa ${ }^{1}$
}

\begin{abstract}
Vancomycin (VCM) has been reported to elicit adverse cutaneous drug reactions. However, VCM-associated purpuric drug eruption has not been reported yet, except leukocytoclastic vasculitis. A 16-year-old Japanese girl was admitted with a respiratory infection. We initiated intravenous administration of VCM. After the start of treatment, impalpable purpuric eruption appeared on her trunk. The eruption gradually extended to her neck, legs, and arms. Skin biopsy showed vasculitis with lymphocyte infiltration in the superficial dermis. A drug lymphocyte stimulation test yielded positive results for VCM. Her cutaneous symptoms rapidly reversed after the withdrawal of VCM. To the best of our knowledge, this is the first reported case of VCM-associated purpuric drug eruption, which differs from leukocytoclastic vasculitis. We recommend that VCM-associated purpuric drug eruption should be considered in the differential diagnosis during the administration of VCM, and a drug lymphocyte stimulation test may be useful for assessment of pathogenesis.
\end{abstract}

Key words: drug lymphocyte stimulation test, Kartagener syndrome, lymphocyte infiltration, lymphocyte transformation test, meropenem

\footnotetext{
From:

Department of Pediatrics, Yamaguchi University Graduate School of Medicine, 1-1-1 Minamikogushi, Ube, Yamaguchi 755-8505, Japan

Department of Dermatology, Yamaguchi University Graduate School of Medicine, 1-1-1 Minamikogushi, Ube, Yamaguchi 755-8505, Japan Department of Diagnostic Pathology, Yamaguchi University Hospital, 1-1-1 Minamikogushi, Ube, Yamaguchi 755-8505, Japan
}

\section{Introduction}

Vancomycin (VCM), a glycopeptide antibiotic, is widely used to treat methicillin-resistant Staphylococcus aureus infections. VCM has been reported to elicit adverse reactions, including red man syndrome, skin rash, and Stevens-Johnson syndrome (SJS). ${ }^{1,2}$ However, there are no reports regarding VCM-associated purpuric drug eruption, except leukocytoclastic vasculitis (LV). We describe the case of a patient with VCM-associated purpuric drug eruption, which differs from LV.

\section{Case Report}

A 16-year-old Japanese girl was admitted with a respiratory infection. She had underlying Kartagener syndrome, hypoplastic left heart syndrome (post-Fontan procedure), and bronchial asthma, which were well controlled. We initiated intravenous administration of VCM and meropenem (MEPM). On day 6 after the start of treatment, an impalpable purpuric eruption appeared on her trunk (Figure 1A). Day 7 laboratory results were as follows: white blood cells, $27.0 \times 10^{9} / \mathrm{L}$ (neutrophil $85.5 \%$, lymphocyte $7.0 \%$, and eosinophil $0.0 \%$ );

\section{Corresponding author:}

Hiroyuki Wakiguchi

Department of Pediatrics, Yamaguchi University Graduate School of Medicine

1-1-1 Minamikogushi, Ube, Yamaguchi 755-8505, Japan

E-mail: hiroyuki@yamaguchi-u.ac.jp

hemoglobin, $9.6 \mathrm{~g} / \mathrm{dL}$; platelet count, $210 \times 10^{9} / \mathrm{L}$; alanine aminotransferase, $18 \mathrm{IU} / \mathrm{L}$ (reference range [rr]: 7-23 IU/L); aspartate aminotransferase, 19 IU/L (rr: 13-30 IU/L); lactate dehydrogenase, 258 IU/L (rr: 124-222 IU/L); and C-reactive protein (CRP), $25.56 \mathrm{mg} / \mathrm{dL}(\mathrm{rr}: \leq 0.14 \mathrm{mg} / \mathrm{dL})$. The eruption became palpable and gradually extended to her neck, legs, and arms (Figure 1B). Skin biopsy showed epidermal liquefaction degeneration, exocytosis, red blood cell infiltration, and single-cell necrosis in the epidermis (Figure 2A). In addition, both hemorrhage and inflammatory cell infiltration, mainly lymphocytes, were observed in the dermis (Figure 2B, 2C). On the other hand, vasculitis with neutrophil infiltration was not observed in the superficial dermis (Figure 2D, 2E). On day 12, VCM and MEPM treatment was changed to teicoplanin and cefozopran. Her cutaneous symptoms gradually improved and disappeared by day 20. Day 20 laboratory results were as follows: white blood cells, $12.0 \times 10^{9} / \mathrm{L}$ (neutrophil $65.0 \%$, lymphocyte $24.0 \%$, and eosinophil $1.5 \%$ ); hemoglobin, $8.6 \mathrm{~g} / \mathrm{dL}$; platelet count, $464 \times 10^{9} / \mathrm{L}$; and CRP, $7.32 \mathrm{mg} / \mathrm{dL}$. We continued treatment, and her CRP levels normalized. A drug lymphocyte stimulation test (DLST; Special Reference Laboratories, Inc., 
APJAI

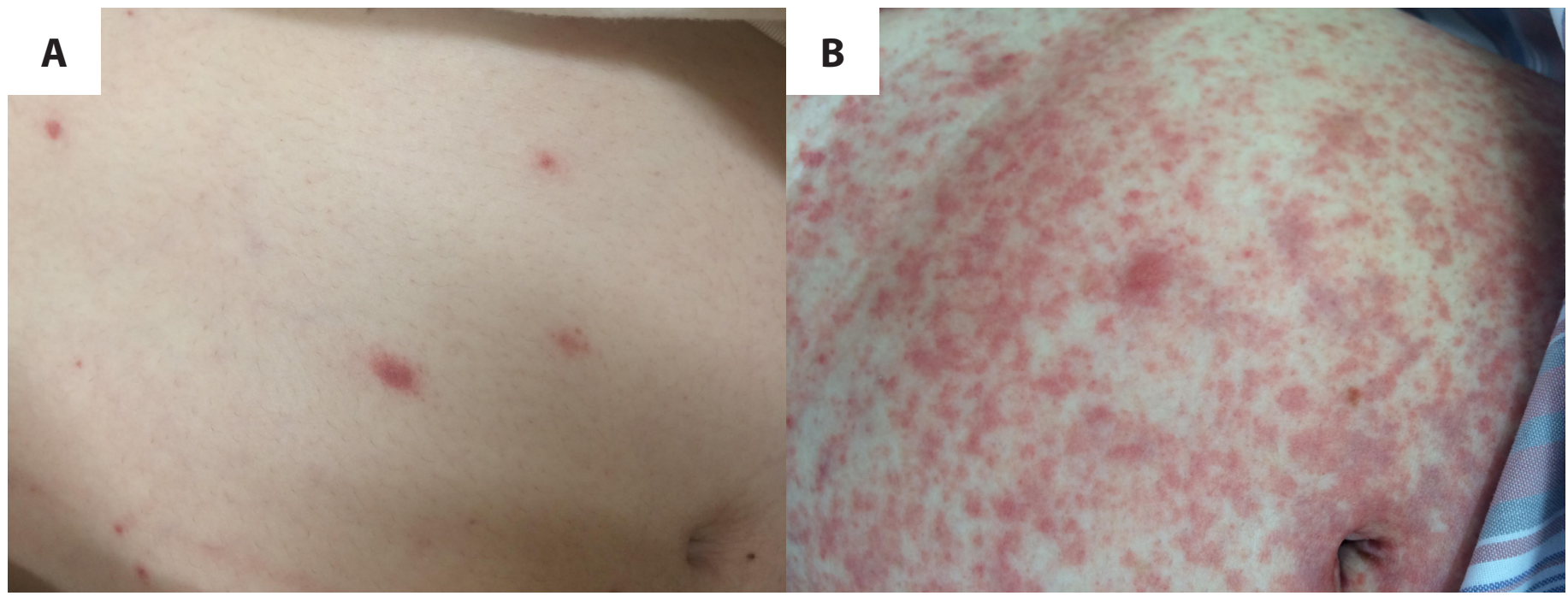

Figure 1. (A) Several purpuras on the patient's abdominal skin are observed. (B) A purpuric eruption gradually extending and coalescing.

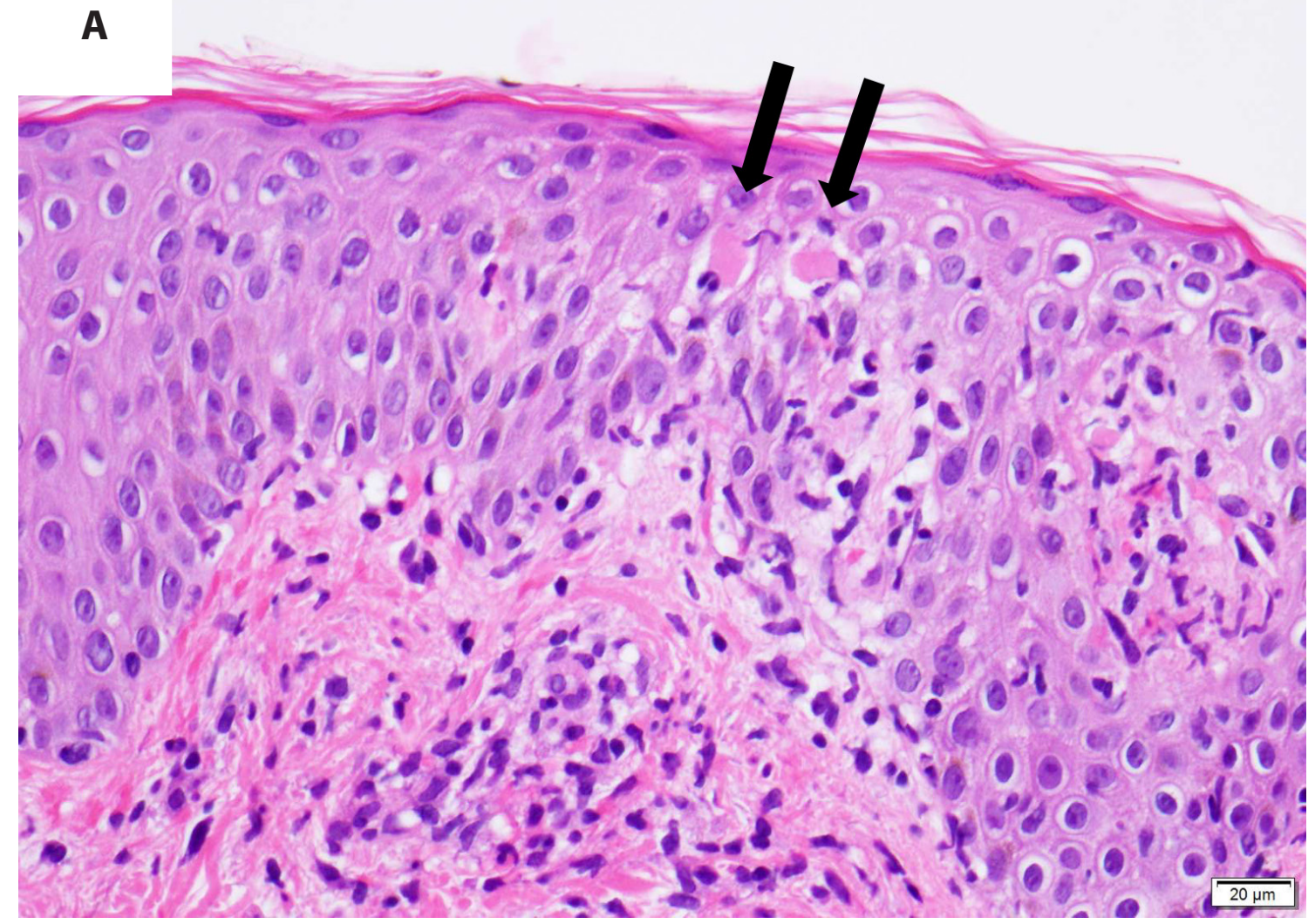

Figure 2. (A) Single-cell necrosis in the epidermis (arrows) and red blood cell infiltration (hematoxylin-eosin [H\&E] staining). 

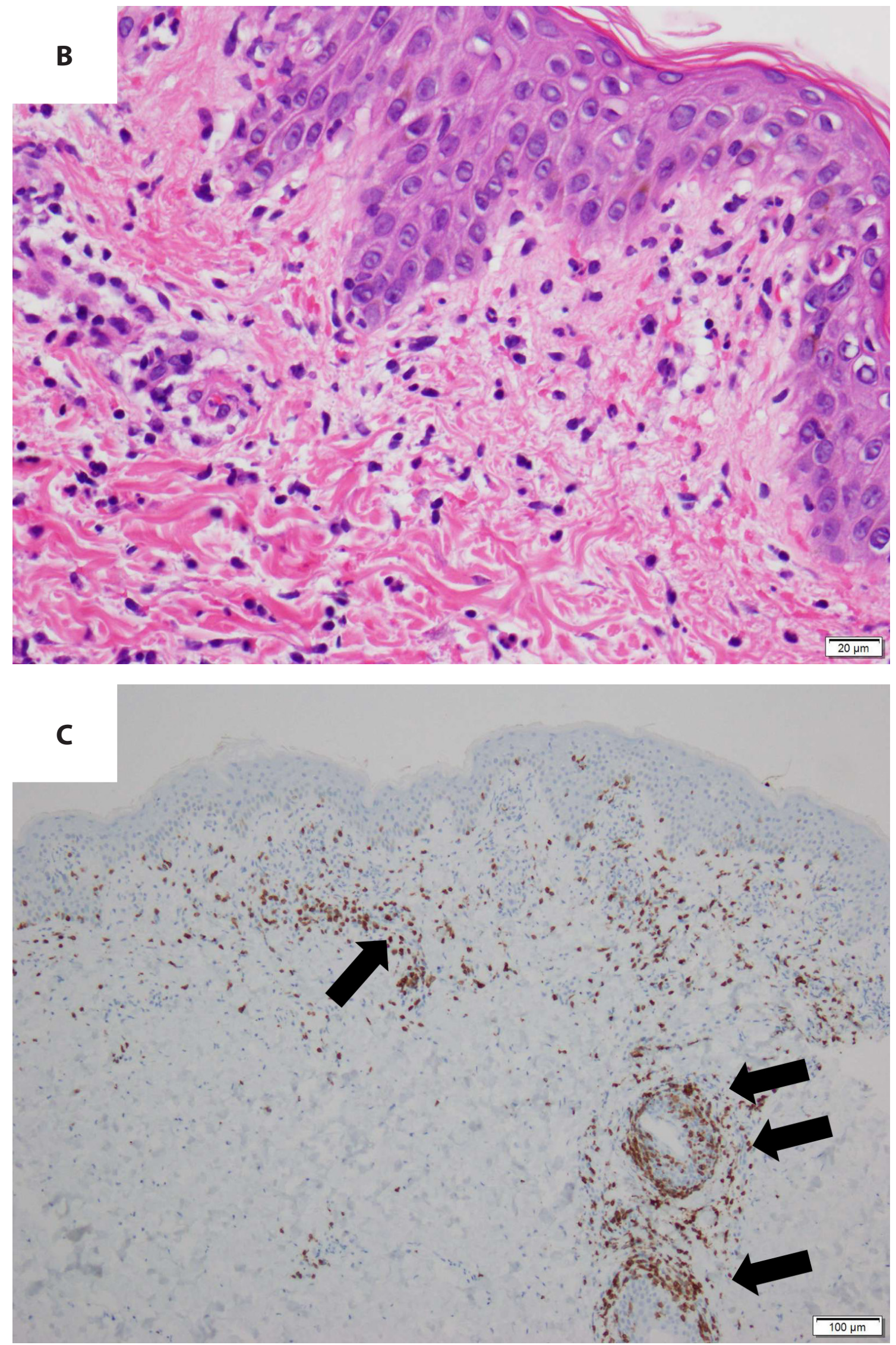

Figure 2. (Continued) (B) Hemorrhage and inflammatory cell infiltration in the dermis (H\&E staining). (C) Lymphocytes are stained not only in the dermis but also in the perivascular lesion (arrows; CD3 staining). 


\section{D}

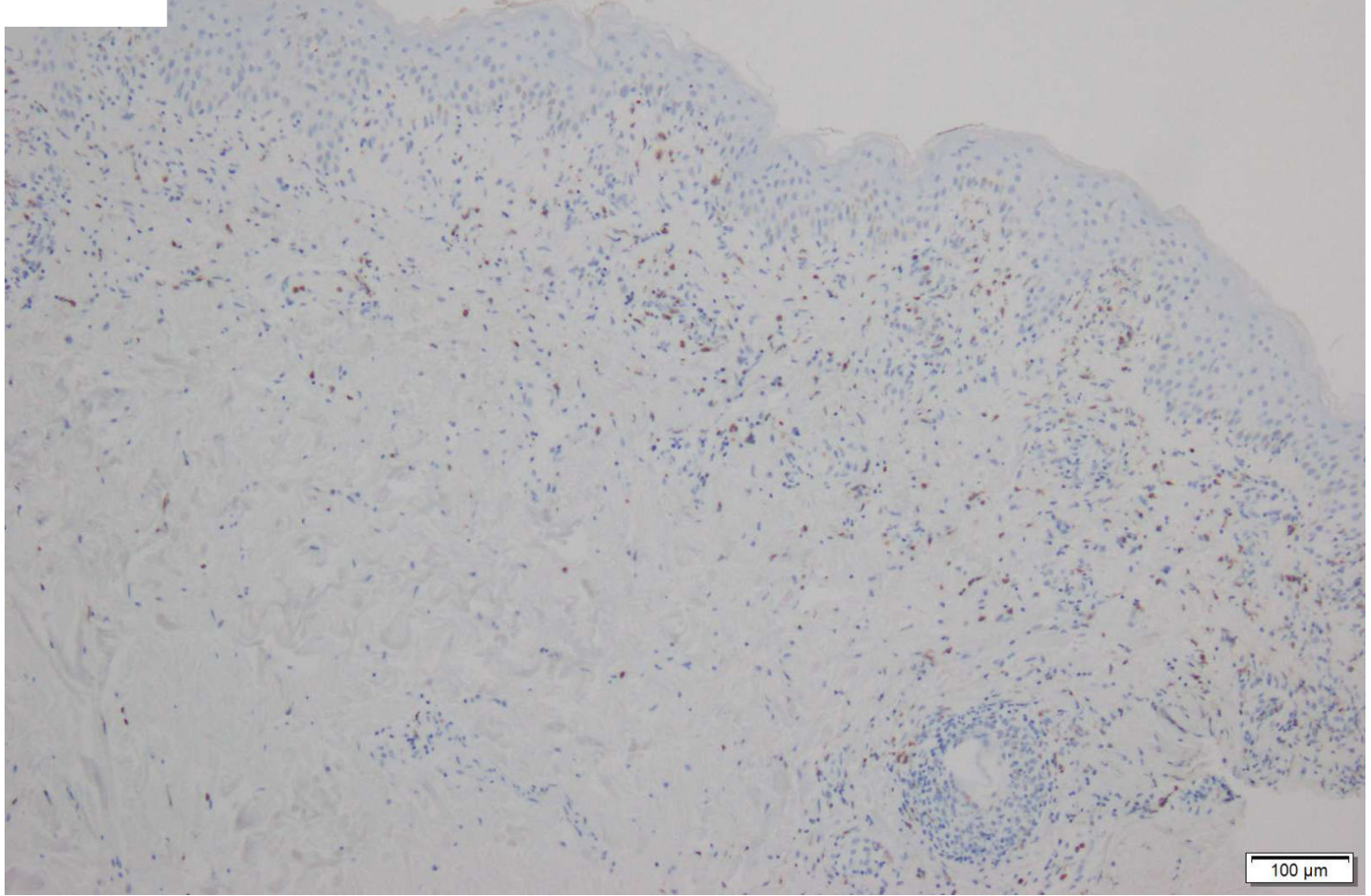

E

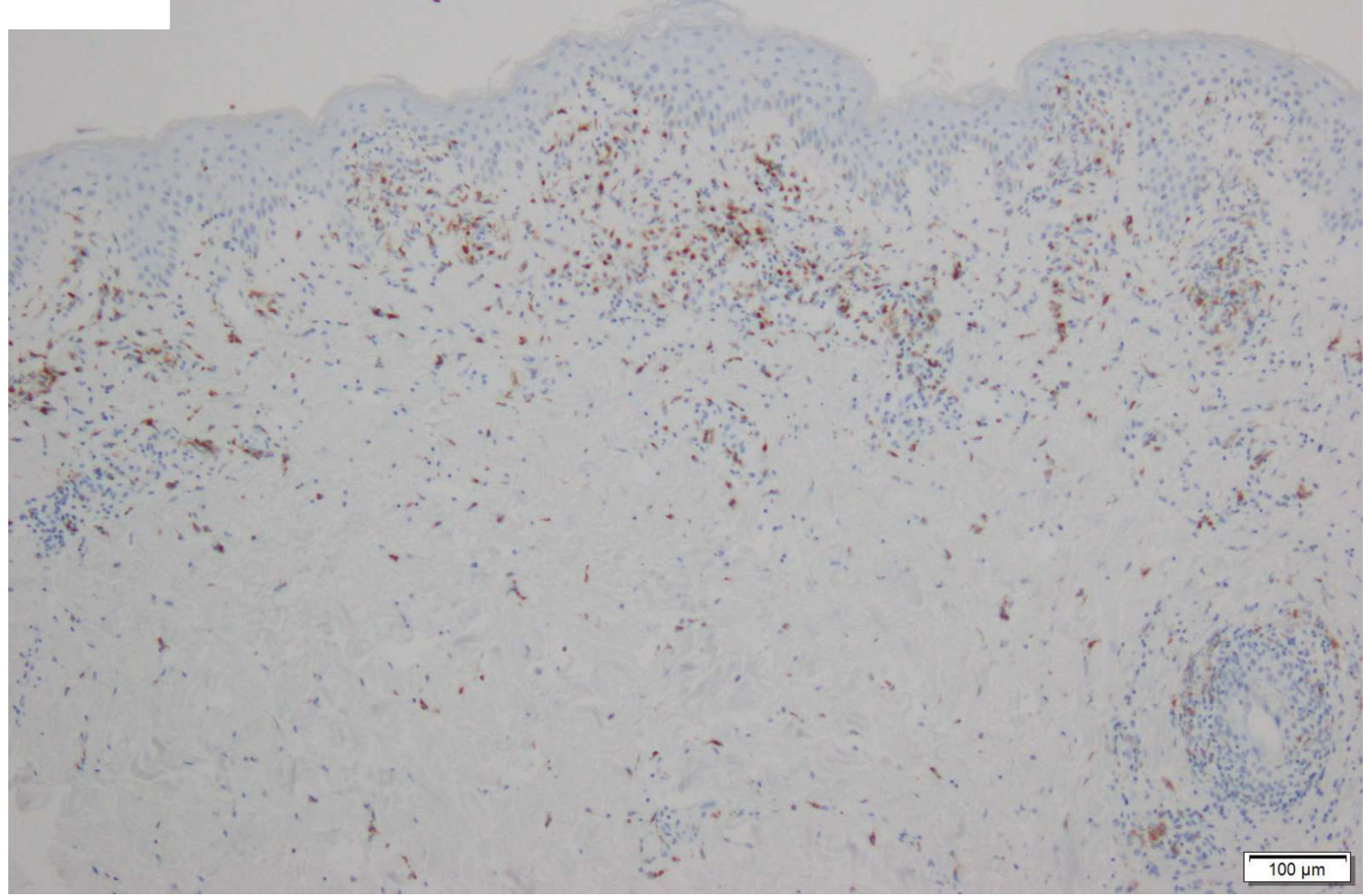

Figure 2. (Continued) (D) A small number of neutrophils are stained mainly in the dermis (myeloperoxidase staining). (E) Histiocytes are stained mainly in the dermis (CD68 staining). 
Tokyo, Japan) using her peripheral mononuclear cells revealed elevated $\left[{ }^{3} \mathrm{H}\right]$ thymidine incorporation with VCM compared with MEPM or a negative control (concentrations of both VCM and MEPM used for the DLST, $250 \mu \mathrm{g} / \mathrm{ml}$; positive control [phytohemagglutinin mitogen], 10,057 cpm; negative control [without drug stimulation], $112 \mathrm{cpm}$ ). The stimulation index of the DLST showed the following results: VCM, 1.89 and MEPM, 1.08 (rr: $\leq 1.80^{3-5}$ for both). Thus, she was considered as having VCM-associated purpuric drug eruption.

\section{Discussion}

Pathological findings showed not only purpura but also some features of erythema multiforme. However, her eruption did not blanch under pressure, and we therefore considered it as purpura. Sometimes, maculopapular drug eruptions exhibit purpuric spots over time. ${ }^{6}$ However, this patient showed purpura from the beginning; thus, we diagnosed her as having purpuric drug eruptions, not maculopapular drug eruptions. Adverse reactions of the skin due to VCM are frequently observed. An et al. reported that skin rashes are the main VCM-induced

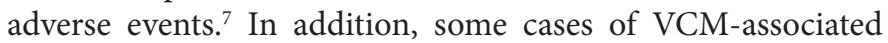
$\mathrm{LV}^{8}$ including Henoch-Schönlein purpura, ${ }^{9}$ both of which are characterized by palpable purpura on the legs with neutrophil infiltration, have been reported. Cases of LV show vasculitis with neutrophil infiltration. ${ }^{10}$ However, the vasculitis in our case mainly comprised the infiltration of lymphocytes, not neutrophils (Figure 2C, 2D, 2E). Reports have described patients with purpuric drug eruption induced by epidermal growth factor receptor inhibitors such as erlotinib, ${ }^{11}$ lenalidomide, ${ }^{12}$ and linezolid. ${ }^{13}$ Neither blood extravasation nor LV was observed in the pathological findings. Conversely, cases of purpura associated with another epidermal growth factor receptor inhibitor, gefitinib-associated LV, have been reported. ${ }^{14}$ However, no previous reports have described purpuric drug eruption without LV that is associated with VCM.

We discontinued the VCM and MEPM due to concerns of VCM-induced SJS, based on the previous report. ${ }^{2}$ Her cutaneous symptoms rapidly reversed after the withdrawal of VCM and MEPM, and no other systemic symptoms or eosinophilia were observed while her purpura disappeared. In addition, the results of a DLST suggested that her eruptions may have been a side effect of VCM. However, we could not perform a drug challenge test because of her general condition. Therefore, the pathogenic role of MEPM cannot be excluded. As the purpura appeared several days after the start of VCM administration, we believe the eruption might have been induced by a delayedtype hypersensitivity reaction.

To the best of our knowledge, this is the first reported case of VCM-associated purpuric drug eruption, which differs from LV. We recommend that VCM-associated purpuric drug eruption should be considered in the differential diagnosis during the administration of VCM, and a DLST may be useful for assessment of pathogenesis.

\section{Acknowledgments}

We thank the patient for her cooperation. We also thank her family and friends.

\section{Conflict of interest}

None.

\section{Financial support}

None.

\section{Informed consent}

Informed consent for publication was obtained from the patient and her guardian.

\section{References}

1. Levy M, Koren G, Dupuis L, Read SE. Vancomycin-induced red man syndrome. Pediatrics. 1990;86:572-80.

2. Alexander II, Greenberger PA. Vancomycin-induced Stevens-Johnson syndrome. Allergy Asthma Proc. 1996;17:75-8.

3. Okamoto O, Fujiwara S. Drug eruption caused by ranitidine hydrochloride (Zantac) which showed a strong reaction in a drug-induced lymphocyte stimulation test. J Dermatol. 2007;1:74-9.

4. Suzuki Y, Miwa S, Shirai M, Ohba H, Murakami M, Fujita K, et al. Drug lymphocyte stimulation test in the diagnosis of adverse reactions to antituberculosis drugs. Chest. 2008;5:1027-32.

5. Sako A, Bae SK, Gushima T, Motoshita J, Bekki S, Abiru S, et al. Drug -induced Liver Injury Associated with Mosapride Citrate: A Report of Two Cases. Intern Med. 2017;1:41-5.

6. Namiki T, Omigawa C, Nojima K, Wakasa T, Tokoro S, Hanafusa T, et al. Case of cetuximab-induced disseminated necrotic and maculopapular eruptions: Involvement of an epidermal growth factor receptor inhibitor with epidermal necrosis. J Dermatol. 2018;1:e3-e4.

7. An SY, Hwang EK, Kim JH, Kim JE, Jin HJ, Jin SM, et al. Vancomycin -associated spontaneous cutaneous adverse drug reactions. Allergy Asthma Immunol Res. 2011;3:194-8.

8. Pongruangporn M, Ritchie DJ, Lu D, Marschall J. Vancomycin-associated leukocytoclastic vasculitis. Case Rep Infect Dis. 2011;2011:356370.

9. Bataille S, Daumas A, Tasei AM, Jourde-Chiche N, Dussol B, Burtey S, et al. Vancomycin-induced Henoch-Schonlein purpura: a case report. J Med Case Rep. 2012;6:106.

10. Elder DE, Elenitsas R, Rosenbach M, Murphy GF, Rubin AI, Xu X, editors. Lever's Histopathology of the Skin. 11th ed. Philadelphia: Wolters Kluwer; 2015.

11. Nakamura-Wakatsuki T, Yamamoto T. Purpuric drug eruption and alopecia induced by erlotinib. Dermatol Online J. 2012;18:16.

12. Kuohung V, Goldberg LJ, Demierre MF. Lenalidomide-induced purpuric eruption: a new adverse cutaneous reaction. J Am Acad Dermatol. 2011;65: 654-6.

13. Kim FS, Kelley W, Resh B, Goldenberg G. Linezolid-induced purpuric medication reaction. J Cutan Pathol. 2009;36:793-5.

14. Uchimiya H, Higashi Y, Kawai K, Kanekura T. Purpuric drug eruption with leukocytoclastic vasculitis due to gefitinib. J Dermatol. 2010;37:562-4. 